\title{
SÍNDROME DE HORNER APÓS CIRURGIA ESTEREOTÁXICA PARA DOENÇA DE PARKINSON
}

\author{
Luiz A. Rogano ', Marushcka Assis', Manoel J. Teixeira'
}

\begin{abstract}
RESUMO - Apresentamos estudo de dez pacientes com doença de Parkison, que foram submetidos a procedimentos ablativos estereotáxicos percutâneos e que desenvolveram síndrome de Horner ipsilateral imediatamente após a lesão. Sete pacientes foram submetidos a palidotomia, dois a subtalamotomia (campotomia de Forel) e talamotomia e um paciente a subtalamotomia. Sete desenvolveram miose e os dez desenvolveram semiptose ipsilateral à lesão. A ocorrência da síndrome de Horner resulta possivelmente de lesão de fibras simpáticas entre o hipotálamo, campo de Forel e tálamo.
\end{abstract}

PALAVRAS-CHAVE: doença de Parkinson, estereotaxia, síndrome de Horner.

Horner syndrome after stereotactic Parkinson's surgery.

ABSTRACT - We present ten patients with Parkinson's disease who underwent stereotactic ablative radiofrequency procedures. Seven patients underwent pallidotomy, two subthalamotomy and VIM, and one subthalamotomy. Seven developed miosis and all semiptosis ipsilateral immediately after the procedure. The occurrence of Horner's syndrome is probably due to the lesion of sympathetic fibers among hypothalamus, Forel's field and thalamus after the stereotactic procedure.

KEY WORDS: Parkinson's disease, stereotactic procedures, Horner's syndrome.

Temos observado o aparecimento da sindrome de Horner (SH) imediatamente após a cirurgia estereotaxica (CE) para doença de Parkinson (DP). A $\mathrm{SH}$ desenvolveu-se ipsilateralmente ao lado submetido a CE. Com o objetivo de se compreender essa fisiopatologia e observar possível ação determinante do aparecimento da SH após CE, foram estudados dez pacientes.

\section{MÉTODO}

No nosso serviço de neurocirurgia, são realizadas anualmente 50 cirurgias estereotáxicas percutâneas por radiofrequencia, para o tratamento da DP. Dez pacientes com quadro de DP refratário a tratamentos clínicos, foram escolhidos aleatoriamente e submetidos sequencialmente a procedimentos ablativos estereotáxicos. Foram medidos os diâmetros pupilares e das fendas palpebrais no pré e pós-operatório imediato dentro do centro cirúrgico. Essas medidas foram feitas sob mesma intensidade de luz e pelo mesmo observador, utilizando escala simples milimetrada. Como a avaliação do diâmetro pupilar e da fenda palpebral, não demanda nenhum procedimento invasivo, e já havia autorização do paciente para a cirurgia estereotáxica, não pedimos novamente consentimento para essa avaliação pupilar e da fenda palpebral.
Todos os doentes foram submetidos a lesão estereotáxica por radiofrequência, guiada por reconstrução de imagem do tálamo, globo pálido e campo de Forel. As reconstruções tridimensionais da imagem dos núcleos da base foram realizadas por tomografia computadorizada com cortes de $2 \mathrm{~mm}$, por programa computadorizado específico (Micromar/Alaminos). Inicialmente os pacientes receberam o halo do aparato de estereotaxia (MicromarTeixeira/São Paulo/Brasil), ao redor do crânio sob anestesia local (Lidocaína $2 \%$ ) e submetidos a tomografia computadorizada. As imagens obtidas foram transportadas para computador, com o programa de reconstrucao de imagem para os cálculos estereotáxicos. Em seguida, os pacientes foram encaminhados ao centro cirúrgico e foram submetidos, com anestesica local, a incisao paramediana retilinea a $3 \mathrm{~cm}$ da linha média e a frente da sutura coronal. Foi realizado orifício de trepanação de $1 \mathrm{~cm}$ de diâmetro e abertura da dura-mater. Com os cálculos estereotáxicos, foi montado o sistema de estereotaxia (Micromar) e introduzido eletrodo para estimulação e coagulação por aparelho de radiofrequência (Radionics, Burlington, USA) com área de exposição de $4 \mathrm{~mm}$ de comprimento por 1,2 $\mathrm{mm}$ de diâmetro. Foi realizada a estimulação das estruturas nervosas variando de 1 a 6 volts e 5 a $100 \mathrm{~Hz}$, e, em concordância com parâmetros de respostas, foram realizadas duas a três lesões sequênciais, com temperatura

Equipe de Neurocirurgia do Hospital 9 de Julho, São Paulo SP, Brasil: ${ }^{1}$ Neurocirurgião.

Recebido 30 Julho 2002, recebido na forma final 11 Novembro 2002. Aceito 16 Novembro 2002.

Dr. Luiz A. Rogano - Rua Dr. Veiga Filho 350/503 - 01229-000 São Paulo SP - Brasil 
Tabela 1. Dados quanto aos pacientes estudados.

\begin{tabular}{|c|c|c|c|}
\hline $\begin{array}{l}\text { Sexo/ } \\
\text { Idade } \\
\text { (anos) }\end{array}$ & Cirurgia & $\begin{array}{l}\text { Pré operatório } \\
\text { Pupila } \\
\text { Fenda }(\mathrm{mm})\end{array}$ & $\begin{array}{l}\text { Pós operatório } \\
\text { Pupila } \\
\text { Fenda (mm) }\end{array}$ \\
\hline $1-M, 51$ & Palidotomia D & $\begin{array}{l}D=3, E=3 \\
D=7, E=7\end{array}$ & $\begin{array}{l}D=3, E=3 \\
D=5, E=7\end{array}$ \\
\hline $2-M, 70$ & Palidotomia D & $\begin{array}{l}D=2, E=3 \\
D=11, E=11\end{array}$ & $\begin{array}{l}D=2, E=3 \\
D=7, E=11\end{array}$ \\
\hline $3-M, 75$ & Palidotomia E & $\begin{array}{l}D=3, E=3 \\
D=8, E=7\end{array}$ & $\begin{array}{l}D=3, E=2 \\
D=8, E=5\end{array}$ \\
\hline $4-M, 75$ & Palidotomia D & $\begin{array}{l}D=5, E=5 \\
D=11, E=11\end{array}$ & $\begin{array}{l}D=4, E=5 \\
D=7, E=11\end{array}$ \\
\hline $5-M, 58$ & Palidotomia D & $\begin{array}{l}D=4, E=4 \\
D=10, E=10\end{array}$ & $\begin{array}{l}D=3, E=4 \\
D=6, E=10\end{array}$ \\
\hline $6-F, 65$ & $\begin{array}{l}\text { SubTalamotomia } \\
+ \text { VIM D }\end{array}$ & $\begin{array}{l}D=3, E=3 \\
D=10, E=10\end{array}$ & $\begin{array}{l}D=2, E=3 \\
D=6, E=10\end{array}$ \\
\hline $7-F, 72$ & Palidotomia D & $\begin{array}{l}D=5, E=5 \\
D=11, E=11\end{array}$ & $\begin{array}{l}D=5, E=5 \\
D=7, E=11\end{array}$ \\
\hline $8-M, 56$ & Palidotomia E & $\begin{array}{l}D=2, E=3 \\
D=12, E=14\end{array}$ & $\begin{array}{l}D=2, E=1 \\
D=12, E=12\end{array}$ \\
\hline $9-M, 51$ & $\begin{array}{l}\text { SubTalamotomia } \\
+ \text { VIM E }\end{array}$ & $\begin{array}{l}D=5, E=5 \\
D=11, E=11\end{array}$ & $\begin{array}{l}D=5, E=4 \\
D=11, E=9\end{array}$ \\
\hline $10-M, 61$ & $\begin{array}{l}\text { Sub } \\
\text { Talamotomia D }\end{array}$ & $\begin{array}{l}D=3, E=3 \\
D=11, E=11\end{array}$ & $\begin{array}{l}D=1, E=3 \\
D=9, E=11\end{array}$ \\
\hline
\end{tabular}

$D$, direito; $E$, esquerdo; $M$, masculino; $F$, feminino; VIM, núcleo ventro intermédio.

variando entre 70 e $80^{\circ} \mathrm{C}$. Os pacientes que apresentavam, como alteração neurológica predominantemente, rigidez foram submetidos a lesão do globo pálido interno (GPI) e os com predomínio de tremor, no núcleo ventro intermédio (VIM) do tálamo e, para casos de tremor e rigidez, a lesão foi realizada no subtálamo (campo de Forel). Os procedimentos cirúrgicos realizados foram: sete palidotomias, uma subtalamo, duas subtalamo associadas com VIM.

\section{RESULTADOS}

Consta da Tabela 1 os dados de pacientes quanto a: tipo de procedimento cirúrgico, medida do diâmetro pupilar e fenda palpebral pré e pós operatórios.

Os resultados mostraram redução do diâmetro pupilar ipsilateral à lesão pela CE em 7 ( de 1 a 2 mm) dos 10 casos. Em todos os casos houve redução dos diâmetros das fendas palpebrais (de 2 a $4 \mathrm{~mm}$ ).

Os resultados obtidos na Paciente 8 mostraram reduções dos diâmetros da pupila e da fenda palpebral. Esta doente teve o lado direito operado por CE por DP, 9 meses antes e já havia redução do diâmetro pupilar e da fenda palpebral em relação ao do lado esquerdo.

\section{DISCUSSÃO}

A síndrome de Claud Bernard-Horner é caracterizada pela destruição de fibras simpáticas que se dirigem para o globo ocular. Manifesta-se, principalmente por miose com o reflexo pupilar preservado, ptose da pálpebra superior, e elevação da pálpebra inferior e congestão conjuntival. A nível central, esta pode ocorrer devido a lesão de fibras simpáticas do nível do subcórtex até a medula espinal cervical alta ${ }^{1,2}$.

$\mathrm{O}$ aparecimento da SH ipsilateral à CE para o tratamento da DP indicaria que houve o comprometimento das fibras simpáticas ao nível dos gânglios da base. Segundo Delmas², há rica interconexão entre fibras simpáticas do hipotálamo com o tálamo e globo pálido. Essas fibras passam, em sua maioria, pela região subtalâmica $(\mathrm{H} 1$ e $\mathrm{H} 2)$ sendo denominadas de alcas lenticulares. Kim e $\mathrm{Umbach}^{3}$ relataram a sua experiência com o aparecimento da $\mathrm{SH}$ ipsilateral a cirugia para a DP e concluiram que o envolvimento da inervação simpática homolateral indica que as fibras entre o hipotálamo e núcleos da base, passando pela região subtalâmica, estariam envolvidas. $\mathrm{O}$ aparecimento da SH poderia ser explicado pela destruição de fibras simpáticas ao nível do subtálamo quando os pacientes fossem submetidos a subtalamotomia e nas lesões do tálamo e do GP, pela diminuição da aferência dessas vias simpáticas ao hipotálamo 4 .

\section{REFERÊNCIAS}

1. Tolosa APM, Canelas HM. Propedêutica neurológica. Ed.2. São Paulo, São Paulo SP, Brasil, Savier 1975.

2. Delmas A. Vias e centros neurais. 7.ed. Rio de Janeiro Guanabara Koogan, 1973.

3. Kim YK, Umbach W. The effects of stereotaxic subthalamotomy on symphathetic tonus. Confinia Neurol 1972;34:156-160.

4. Kruszewszki YK, Antonoci F, Bordini C, Wester K, Sjaastad O. Sympathetic functions in parkinsonism treated with stereotatic surgery: observations in ten patients. Funct Neurol 1991;6:263-268. 